\title{
Intense pulsed light for the treatment of Meibomian gland dysfunction: A systematic review and meta-analysis
}

\author{
SHUANG LIU, SHAOHUA TANG, HONG DONG and XIAOHAN HUANG \\ Department of Ophthalmology, Beijing Jishuitan Hospital, Beijing 100035, P.R. China
}

Received November 20, 2019; Accepted May 7, 2020

DOI: $10.3892 /$ etm.2020.8838

\begin{abstract}
Meibomian gland dysfunction (MGD) is frequently encountered by eye care practitioners. It is characterised by obstruction of the Meibomian glands and/or alterations in the consistency of glandular secretions. At present, no definitive treatment exists for this condition. The present meta-analysis was performed to assess the efficacy of intense pulsed light (IPL) therapy in the management of MGD. Databases including EMBASE, PubMed, Cochrane Central, MEDLINE and Google Scholar were systematically searched to identify clinical trials that assessed the efficacy of IPL in the treatment of MGD. Outcome measures were described as the standardized mean difference (SMD). The fixed- or random-effects model was selected for analysis based on the Cochrane $\mathrm{I}^{2}$ values representing heterogeneity. Publication bias was visually inspected using Begg's funnel plot. Data were synthesized from four randomized controlled trials (RCTs) comprising 122 subjects in the IPL group and 120 subjects in the control group. Pooled analysis indicated no statistically significant difference in the Standard Patient Evaluation of Eye Dryness (SPEED) scores between the two groups [SMD -0.16 (95\% CI, -0.41 to 0.10)] but a significant increase in Non-Invasive Tear Break-Up Time (NIBUT) scores in the IPL group (SMD, 0.90; 95\% CI, $0.40-1.40)$. To conclude, the results of the present study did not provide any conclusive evidence for the efficacy of IPL therapy in the management of MGD. The analysis indicated that IPL therapy may result in an improvement of objective NIBUT scores but has no effect on subjective SPEED scores. Given the limited number of studies performed to date, there is a requirement for more well-designed prospective RCTs with a larger sample size to provide further evidence on the efficacy of IPL therapy.
\end{abstract}

Correspondence to: Dr Shuang Liu, Department of Ophthalmology, Beijing Jishuitan Hospital, 68 Huinan North Road, Beijing 100035, P.R. China

E-mail: 18611821868@163.com

Key words: dry eye disease, intense pulsed light, Meibomian gland dysfunction, Meibomian gland expression, meibum

\section{Introduction}

Meibomian gland dysfunction (MGD) is a clinical condition that is commonly encountered by ophthalmologists (1). The worldwide incidence varies from $3.5 \%$ to as high as $70 \%$, depending on the geographical region (2-5). Affected patients usually present with symptoms of ocular burning, irritation, red and watery eyes, as well as variation of visual acuity (1). The underlying pathology involves blockage of the terminal ducts of Meibomian glands with or without qualitative/quantitative changes in their glandular secretion $(1,6)$. MGD is characterized by acinous atrophy, hyperkeratinisation of the ductal epithelium and increased viscosity of the meibum. This leads to reduced glandular secretion and encourages growth of commensal bacteria. Lipase secreted by these bacteria brings about changes in the composition and melting point of meibum lipids, which further inhibits glandular output. The reduced secretion of lipids thereby leads to evaporative dry eye, hyperosmolarity and tear film instability $(1,4,7,8)$.

Management of MGD is still empirical and studies have evaluated a wide range of interventions with limited benefits. These include conservative measures such as hot fomentation and lid hygiene, as well as the use of steroidal or non-steroidal anti-inflammatory agents and antibiotics (7-9). Novel techniques, including meibomian gland probing $(10,11)$, intranasal neurostimulation (12), intense pulsed light (IPL) (13-15) and application of electronic heating devices (16), have also been evaluated, but data on their efficacy are limited. Research is still ongoing to identify promising treatment strategies for MGD that are not only efficacious but also have a reasonable safety profile and are acceptable for the patients (15-17).

IPL therapy is not new to the medical field, as it has been widely used in dermatology for the management of a range of conditions, including hypertrichosis, hemangiomas, venous malformations and port-wine stains $(18,19)$. IPL works on the principle of applying a high-intensity non-laser light source that produces wavelengths ranging from 1,500 to $1,200 \mathrm{~nm}$ (19). On application to the skin, the oxyhemoglobin in the red blood cells absorbs the light, resulting in the generation of heat, which activates the coagulation process leading to thrombosis of blood vessels $(17,19)$. The rationale that supports IPL as a potential treatment strategy for MGD is that it has predominant effects to accelerate thrombosis of abnormal blood vessels, liquefaction of meibum leading to its improved secretion, reduced growth and proliferation of pathogenic bacteria and 
reduction in epithelial turnover (18). Apart from these major effects, the procedure also initiates regional photomodulatory effects, activates the fibroblasts and leads to enhancement of collagen synthesis (18). A combination of these mechanisms leads to improvements of the disease condition.

In the past decade, a number of studies have investigated the efficacy of IPL in the management of MGD (20-31). However, these standalone studies vary in terms of study design and methodology. For routine use of IPL therapy in clinical practice, there is a need for high-quality evidence. To the best of our knowledge, there has been no previous study attempting to generate high-level evidence for IPL therapy in MGD in the form of a systematic review and meta-analysis. Therefore, the purpose of the present study was to systematically review the literature and perform a meta-analysis to assess the efficacy of IPL therapy in the management of MGD.

\section{Materials and methods}

Search strategy. A thorough and systematic search was performed in the EMBASE, PubMed, Cochrane Central, MEDLINE and Google Scholar databases. Randomized controlled trials (RCTs) evaluating the efficacy of IPL in the management of MGD were identified. Relevant studies published since the year 1950 were eligible for inclusion in the meta-analysis. The search was confined until July 2019. A systematic search strategy was developed using the key terms 'Meibomian gland disease', 'intense pulse light' and 'dry eye disease'. There were no limitations regarding the language and only studies on human subjects were eligible to be included. The bibliographies of eligible articles were reviewed to identify additional studies of relevance to this meta-analysis. The Preferred Reporting Items for Systematic review and Meta-Analysis guidelines were followed during the conduct of this review (32).

Selection criteria and methods. A total of two authors (ST and HD) independently reviewed citations and selected studies. After initial screening of titles and abstracts and removal of duplicates, the full-text articles of potentially suitable studies were reviewed. Any discrepancies pertaining to the inclusion of studies were resolved through discussion among the authors of the present study.

Inclusion criteria. For a study to be included in the meta-analysis, it was required to be an RCT comparing the effect of IPL therapy for MGD with a control. The study was required to include a population of only MGD with pre-defined diagnostic criteria and to evaluate at least one of the following outcome measures: Standard Patient Evaluation of Eye Dryness (SPEED) score, tear break-up time (TBUT) and Non-Invasive Tear Break-Up Time (NIBUT) score.

Exclusion criteria. Studies were excluded if they did not provide extractable data on effect sizes (such as mean and standard deviations for the score change) for relevant outcomes and if they were non-randomized studies such as case reports, observational studies (case-control, cohort studies) or review articles. Studies including patients with a history of any ocular surgery and/or those that used contact lenses, as well as patients who were not followed up at future time-points, were also excluded.

Data extraction. Extraction of relevant data from included studies was performed by two authors (HD and XH) independently using a structured data extraction sheet. The following data were extracted from the eligible studies: Surname of first author, year of publication, country, study design, number of MGD participants treated by IPL and placebo, mean age of patients in the IPL and placebo group, sex distribution, type of intervention and comparator, scale parameters measured (including SPEED and NIBUT), IPL intensity, IPL machine used and follow-up duration.

Quality assessment. The methodological assessment was performed by two authors (HD and $\mathrm{XH}$ ) independently and any disagreements were resolved by discussion among all of the authors. The Cochrane Collaboration risk of bias assessment tool for RCTs was used for quality assessment of the included trials (33). Studies were rated as low, high or unclear risk of bias for: Random sequence generation, allocation concealment, blinding of participants and personnel, blinding of outcome assessment, incomplete outcome data, selective reporting and other biases.

Statistical analysis. Statistical analysis was performed using Review Manager [RevMan, version 5.3; Nordic Cochrane Centre (Cochrane Collaboration)]. Pooled estimates for the continuous outcome measures were reported as the standard mean difference (SMD) with 95\% CI. The SMD was generated using the mean and standard deviation (SD) reported in the individual RCTs included in the meta-analysis. $\mathrm{P}<0.05$ was considered to indicate statistical significance. Heterogeneity was examined using Tau and Higgins' $\mathrm{I}^{2}$ and it governed whether a fixed-effects model ( $\mathrm{I}^{2}$ value $\left.\leq 50 \%\right)$ or a random-effects model ( $\mathrm{I}^{2}$ value $>50 \%$ ) was used. Begg's funnel plot was used to visually inspect the presence or absence of publication bias.

\section{Results}

Identification of relevant studies. Fig. 1 presents a flowchart of the literature search and indicates the process through which the selection of articles was made for inclusion in the present review. Using the pre-defined search strategy, a total of 131 published articles were identified. Out of these, only 4 RCTs were finally included in the review. The reasons for exclusion of studies were as follows: Not relevant to the topic $(n=98)$, duplicate records $(n=14)$, studies with MGD but without IPL therapy $(n=2)$, observational studies $(n=10)$, and full text not available in English language $(n=1)$.

Characteristics of the studies. The primary characteristics of the studies included in the present systematic review and meta-analysis are listed in Table I. Overall, four RCTs were included in the meta-analysis, including 122 subjects with MGD and 120 control subjects. All of the included studies had obtained approval from their institutional ethical committee and followed the Declaration of Helsinki guidelines for research on human subjects. The total sample size in the included studies ranged from 20 to 44 participants. In all 


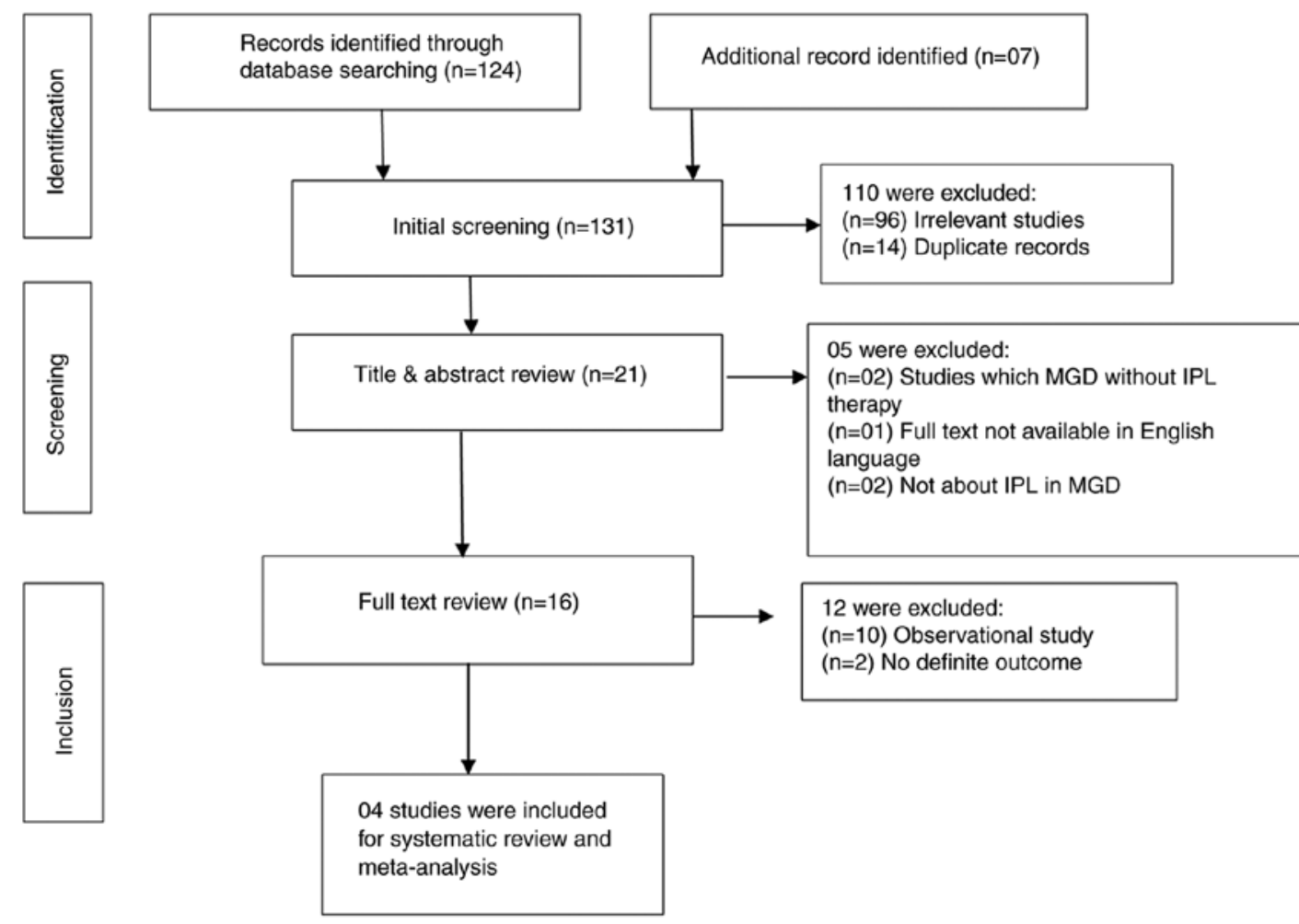

Figure 1. Flow diagram of the selection of studies and specific reasons for exclusion from the present meta-analysis. IPL, intense pulsed light; MGD, Meibomian gland dysfunction.

studies included, data for only two commonly used scales, i.e. SPEED and NIBUT score, were reported. These scores were reported as the mean with SD; they were utilized in the pooling of studies and subsequent calculation of effect sizes with $95 \% \mathrm{CI}$. The IPL intensity ranged from 9 to $17 \mathrm{~J} / \mathrm{cm}^{2}$. The follow-up ranged from 45 days to 9 months (Table I).

Methodological quality. The quality of the individual studies is presented in Table II. Adequate method of randomization and allocation concealment were described in three trials $(20,24,25)$. Blinding of participants and outcome assessment was employed by three studies $(20,24,25)$. Attrition bias was high in one study (25). Only two studies were prospectively registered $(24,25)$.

Meta-analysis. Data were synthesized from four RCTs employing IPL therapy for the treatment of MGD, including 122 subjects in the IPL arm and 120 subjects in the control arm. The results indicated a non-significant reduction of MGD symptoms after IPL therapy based on the SPEED score (SMD, -0.16 ; $95 \%$ CI, -0.41 to 0.10 ; Fig. 2A). However, there was a statistically significant increase in the NIBUT score with IPL therapy compared to the control arm (SMD, 0.90; 95\% CI, 0.40 to 1.40 ; Fig. 2B). Begg's funnel plots were used to assess potential publication bias of the included studies. The shapes of the funnel plots suggested absence of publication bias (Fig. 3A and B).

Salient features of the included studies. The study by Craig et al (20) from 2015 was a prospective placebo-controlled trial comprised of MGD patients. The study indicated a substantial improvement in NIBUT, lipid layer grading and tear film quality, as well as a decline in the SPEED score provided by IPL treatment. The key strength of the study was that only one eye of the participant was subjected to IPL treatment, while the other was used as a control; this allowed for direct comparison between the treatment group and the control group without the requirement for documenting and adjusting for confounders. However, the study also had various limitations. First, the authors did not provide a comprehensive description of the inclusion criteria and the participant selection method. Furthermore, the study did not provide a comprehensive description of the participant characteristics, which curtailed the external validity of the results of the study. In addition, the study indicated that the results obtained were cumulative in nature and therefore, since the data collection was stopped at only day 45 , the full benefit of the treatment may not have been realized. As another limitation, the lack of results in terms of SPEED scores may have resulted from the short duration of the study and a certain benefit may have been identified with a longer duration. Finally, the control eye was exposed to light but had a white light blocker to protect it. A more effective method would likely have been to block all light or use no light at all, as it is possible that a certain amount of light may have passed through the blocker. Besides these issues, the authors did not perform any power calculations to support that the sample size was adequate.

The study by Rong et al (25) from 2018 reported a significant improvement of Meibomian gland function in the eyes with experimental treatment compared to the control eyes in the same patients, that received sham IPL treatment. The intervention and control eyes received Meibomian gland 


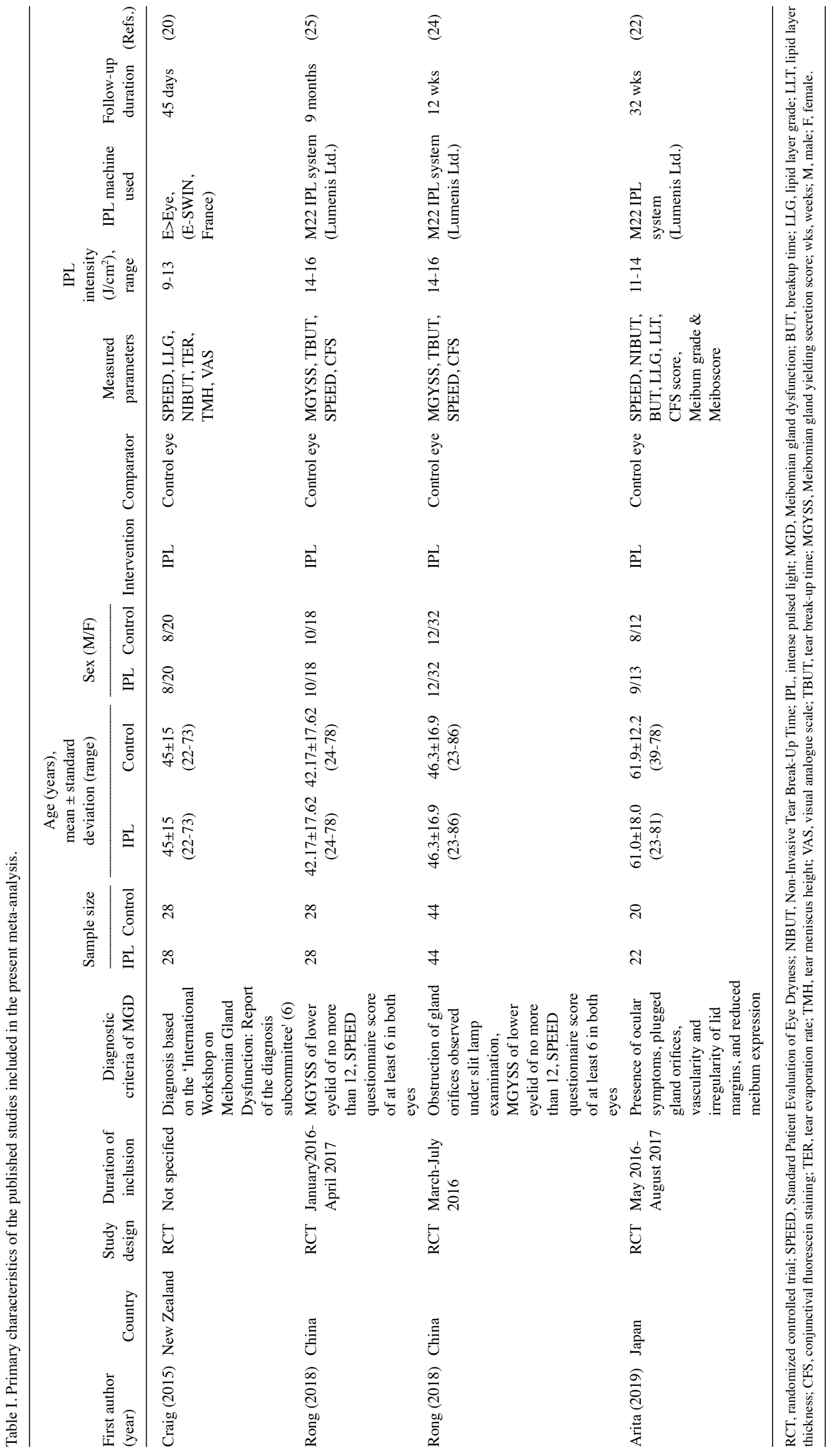


Table II. Quality assessment of the studies included in the meta-analysis.

\begin{tabular}{|c|c|c|c|c|c|c|c|}
\hline Study & $\begin{array}{l}\text { Random sequence } \\
\text { generation }\end{array}$ & $\begin{array}{l}\text { Allocation } \\
\text { concealment }\end{array}$ & $\begin{array}{c}\text { Blinding of participants } \\
\text { and personnel }\end{array}$ & $\begin{array}{c}\text { Blinding of } \\
\text { outcome assessment }\end{array}$ & $\begin{array}{c}\text { Incomplete } \\
\text { outcome data }\end{array}$ & $\begin{array}{l}\text { Selective } \\
\text { reporting }\end{array}$ & Other bias \\
\hline Craig et al (20), 2015 & Low risk & Low risk & Low risk & Low risk & Low risk & Unclear risk & Low risk \\
\hline Rong et al (25), 2018 & Low risk & Low risk & Low risk & Low risk & High risk & Low risk & Low risk \\
\hline Rong et al (24) 2018 & Low risk & Low risk & Low risk & Low risk & Low risk & Low risk & Low risk \\
\hline Arita et al (22), 2019 & Unclear risk & Unclear risk & Unclear risk & Unclear risk & Low risk & Unclear risk & Unclear risk \\
\hline
\end{tabular}

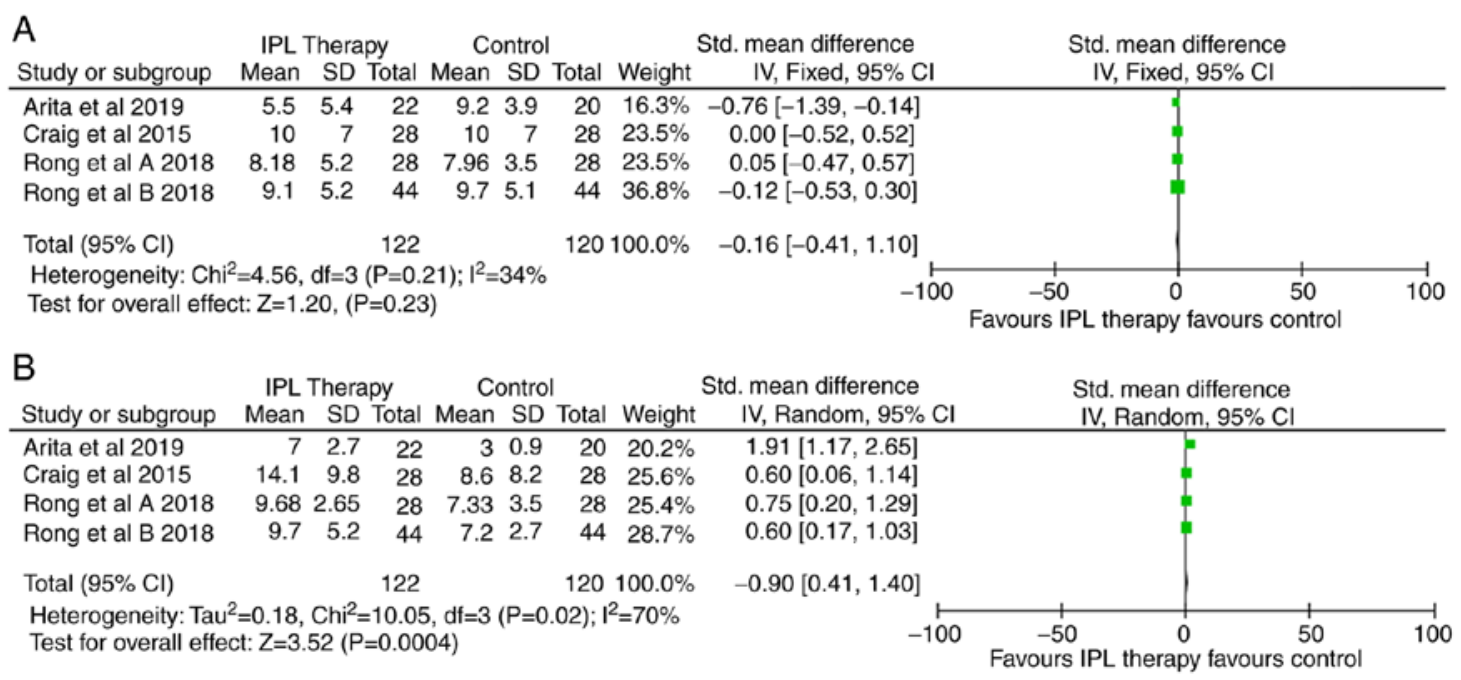

Figure 2. Forest plot for the IPL therapy in patients with Meibomian gland dysfunction. (A) Standard Patient Evaluation of Eye Dryness score change and (B) Non-Invasive Tear Break-Up Time score change. IPL, intense pulsed light; SD, Std. deviation; Std., standard; IV, inverse variance; df, degrees of freedom.
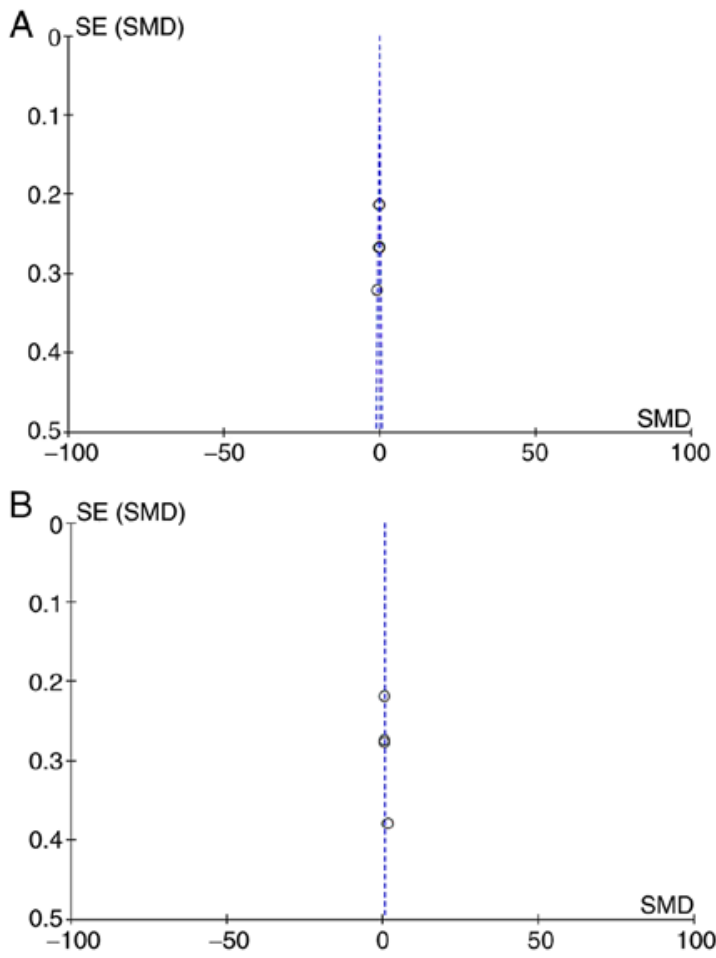

Figure 3. Begg's funnel plot for the studies included in the meta-analysis for intense pulsed light therapy in patients with Meibomian gland dysfunction. (A) Standard Patient Evaluation of Eye Dryness score change and (B) Non-Invasive Tear Break-Up Time score change. IPL, intense pulsed light; MGD, Meibomian gland dysfunction; SE, standard error; SMD, standard mean difference. expression (MGX) and artificial tears. The study demonstrated that IPL treatment applied directly on the eyelids provided sustained relief to patients with MGD for at least 6 months by improving the secretion function of the Meibomian gland, increasing TBUT and improving ocular symptoms. In the eye receiving IPL, the Meibomian gland yielding secretion score (MGYSS) of the upper as well as the lower eyelid significantly improved at 1,3 and 6 months after the treatment. The study had a high loss to follow-up rate. Out of the 44 participants initially enrolled in the trial, only 28 completed the assessments (i.e. 63.6\%). This high attrition rate may have affected the representativeness of the study sample.

The study by Rong et al (24) from 2018 documented that IPL treatment on the eyelids combined with MGX provided sustained relief for at least 6 months to MGD patients by improving Meibomian gland secretion function, increasing TBUT and improving symptoms. The MGYSS and of the upper and lower eyelid significantly improved in the eyes subjected to IPL treatment at 1, 3 and 6 months after therapy. The study revealed significantly greater improvements in Meibomian gland secretory function in the lower eyelids as compared with those in the upper eyelids. There were certain limitations to this study that require mentioning. The majority of study participants were females, which may affect the representativeness and external validity of the results. Furthermore, the treatment outcomes may have been influenced due to the study investigators having chosen a relatively fixed treatment energy of $14-16 \mathrm{~J} / \mathrm{cm}^{2}$. 
In 2019, Arita et al (22) reported on a prospective multicentre randomized trial demonstrating that a series of IPL-MGX treatment sessions significantly improved the symptoms and signs of refractory MGD as compared with MGX alone. The outcomes of the study pertained to Meibomian glands and the lipid layer of the tear film and these parameters were measured at each of the treatment sessions $(n=8$ in total) prior to the start of treatment and thereafter, and further, for up to 11 weeks after the final treatment. A limitation of the study was insufficient power due to the low number of enrolled patients. Although not a limitation per se, the study did not attempt to present the mechanisms underlying the effectiveness of IPL-MGX treatment.

\section{Discussion}

The present study is novel as it is, to the best of our knowledge, the first meta-analysis that performed a data synthesis of available evidence on the efficacy of IPL therapy in the management of MGD. However, the results of this meta-analysis are inconclusive regarding the benefit of IPL therapy in alleviating the symptoms of MGD. The outcomes primarily considered in the included studies were the NIBUT and SPEED scores. No significant differences in SPEED scores between the two study groups were obtained, but there was a statistically significant difference in the NIBUT scores favoring treatment with IPL therapy.

The observations derived from previous studies indicate that the mechanism of action of IPL may have a neurological basis, as flash application to temporal and lower eyelid regions has been indicated to stimulate branches of parasympathetic nerve that initiates normal activity of the Meibomian gland. Furthermore, by causing thrombosis of abnormal telangiectatic blood vessels, decreasing the levels of inflammatory markers and curbing the proliferation of an abnormal bacterial flora around the eyelids, IPL therapy appears to alleviate the symptoms of MGD. Published studies, mostly observational in nature, evaluating the effectiveness of IPL in the treatment of MGD have reported improvements in the oil flow score (34), tear film osmolarity (17), redness and vascularity (34,35), Meibomian gland expression (26,35), Meibum viscosity and secretion quality $(26,34,35)$, corneal fluorescein staining (17,35), lid margin edema (28) and conjunctival injection $(26,35)$.

The present analysis was based on two important outcome measures (SPEED and NIBUT). The SPEED questionnaire is an easy to use, repeatable, valid and subjective tool to quantify the symptoms of a patient with dry eye disease (36). Other objective techniques of dry eye assessment included TBUT and NIBUT (37). TBUT is an invasive method requiring instillation of fluorescein solution in the eye, which disturbs the tear film equilibrium, causing increased evaporation and tear film destabilization. Given the reduced sensitivity and specificity of TBUT, non-invasive methods of tear film assessment, including NIBUT, have been developed, which do not require instillation of fluorescein. NIBUT is considered to be more precise in assessing tear film stability compared with TBUT (37). In the present analysis, no significant difference in the SPEED scores between the IPL and control group was obtained. This may be attributed to the lack of significant differences in SPEED scores in three of the four studies included in the present review. Only the trial by Arita et al (22) reported significantly reduced SPEED scores in the IPL therapy group. The lack of a statistically significant difference in SPEED scores in the present analysis may be attributed to use of artificial teardrops in the control group of certain trials $(24,25)$, which may have influenced this subjective outcome. However, the present results demonstrated a significant increase in the NIBUT values in the IPL group as compared with those in the control group, suggesting the potential benefit of IPL therapy in the management of MGD. Reduced NIBUT scores are characteristic for patients with MGD and the increase in NIBUT values confirms the role of IPL in improving tear film stability.

The strengths of the present review include a lack of publication bias, confirming the validity of the results. The present results provide important clues on which future research may be based. Continued efforts to perform research studies, particularly RCTs, are necessary to further establish the efficacy of IPL therapy as a treatment option in the management of MGD.

The present meta-analysis also has certain limitations. The participants in the included studies were predominantly female adults and therefore, the results are likely to relate to this population, thereby limiting the external validity. Secondly, the present meta-analysis was limited to only NIBUT and SPEED scores and other outcome measures such as MGYSS and corneal fluorescein staining could not be analyzed. Finally, the limited number of studies with small sample size included in the present analysis limited the possibility to draw strong conclusions.

In conclusion, the results of the present study did not provide conclusive evidence for the benefit of IPL therapy in the management of MGD. The present analysis indicates that IPL therapy may result in an improvement of objective NIBUT scores but has no significant effect on subjective SPEED scores. Given the limited number of studies performed to date, there is a requirement for more well-designed prospective RCTs with a larger sample size to provide further evidence on the efficacy of IPL therapy.

\section{Acknowledgements}

Not applicable.

\section{Funding}

This study was supported by the Beijing Talents Fund (grant no. 2015000021467G176).

\section{Availability of data and materials}

The datasets used and/or analyzed during the current study are available from the corresponding author on reasonable request.

\section{Authors' contributions}

SL designed the study. ST, HD and XH were involved in the literature search and data interpretation. ST was responsible for data analysis. SL prepared the manuscript. XH edited the manuscript. All authors read and approved the final manuscript. 


\section{Ethical approval and consent to participate}

Not applicable.

\section{Patient consent for publication}

Not applicable.

\section{Competing interests}

The authors declare that they have no competing interests.

\section{References}

1. Bron AJ, Benjamin L and Snibson GR: Meibomian gland disease. Classification and grading of lid changes. Eye (Lond) 5: 395-411, 1991.

2. Lekhanont K, Rojanaporn D, Chuck RS and Vongthongsri A: Prevalence of dry eye in Bangkok, Thailand. Cornea 25 1162-1167, 2006

3. Lin PY, Tsai SY, Cheng CY, Liu JH, Chou P and Hsu WM: Prevalence of dry eye among an elderly Chinese population in Taiwan: The Shihpai Eye Study. Ophthalmology 110: 1096-1101, 2003.

4. Uchino M, Dogru M, Yagi Y, Goto E, Tomita M, Kon T, Saiki M, Matsumoto Y, Uchino Y, Yokoi N, et al: The features of dry eye disease in a Japanese elderly population. Optom Vis Sci 83: 797-802, 2006.

5. Jie Y, Xu L, Wu YY and Jonas JB: Prevalence of dry eye among adult Chinese in the Beijing Eye Study. Eye (Lond) 23: 688-693, 2009.

6. Tomlinson A, Bron AJ, Korb DR, Amano S, Paugh JR, Pearce EI, Yee R, Yokoi N, Arita R and Dogru M: The international workshop on meibomian gland dysfunction: Report of the diagnosis subcommittee. Invest Ophthalmol Vis Sci 52: 2006-2049, 2011.

7. Dougherty JM, McCulley JP, Silvany RE and Meyer DR: The role of tetracycline in chronic blepharitis. Inhibition of lipase production in staphylococci. Invest Ophthalmol Vis Sci 32: 2970-2975, 1991.

8. Tabbara KF, al-Kharashi SA, al-Mansouri SM, al-Omar OM, Cooper H, el-Asrar AM and Foulds G: Ocular levels of azithromycin. Arch Ophthalmol 116: 1625-1628, 1998.

9. Schultz C: Safety and efficacy of cyclosporine in the treatment of chronic dry eye. Ophthalmol Eye Dis 6: 37-42, 2014

10. Ma X and Lu Y: Efficacy of intraductal meibomian gland probing on tear function in patients with obstructive meibomian gland dysfunction. Cornea 35: 725-730, 2016.

11. Sik Sarman Z, Cucen B, Yuksel N, Cengiz A and Caglar Y: Effectiveness of intraductal meibomian gland probing for obstructive meibomian gland dysfunction. Cornea 35: 721-724, 2016.

12. Gumus K, Schuetzle KL and Pflugfelder SC: Randomized controlled crossover trial comparing the impact of sham or intranasal tear neurostimulation on conjunctival goblet cell degranulation. Am J Ophthalmol 177: 159-168, 2017.

13. Raulin C, Greve B and Grema H: IPL technology: A review. Lasers Surg Med 32: 78-87, 2003.

14. Babilas P, Schreml S, Szeimies R-M and Landthaler M: Intense pulsed light (IPL): A review. Lasers Surg Med 42: 93-104, 2010.

15. Schuh A, Priglinger S and Messmer EM: Intense pulsed light (IPL) as a therapeutic option for Meibomian gland dysfunction. Ophthalmologe 116: 982-988, 2019 (In German).

16. Brinton M, Kossler AL, Patel ZM, Loudin J, Franke M, Ta CN and Palanker D: Enhanced tearing by electrical stimulation of the anterior ethmoid nerve. Invest Ophthalmol Vis Sci 58: 2341-2348, 2017

17. Toyos R, McGill W and Briscoe D: Intense pulsed light treatment for dry eye disease due to meibomian gland dysfunction; a 3-year retrospective study. Photomed Laser Surg 33: 41-46, 2015.
18. Dell SJ, Gaster RN, Barbarino SC and Cunningham DN: Prospective evaluation of intense pulsed light and meibomian gland expression efficacy on relieving signs and symptoms of dry eye disease due to meibomian gland dysfunction. Clin Ophthalmol 11: 817-827, 2017.

19. Goldberg DJ: Current trends in intense pulsed light. J Clin Aesthet Dermatol 5: 45-53, 2012.

20. Craig JP, Chen YH and Turnbull PR: Prospective trial of intense pulsed light for the treatment of meibomian gland dysfunction. Invest Ophthalmol Vis Sci 56: 1965-1970, 2015.

21. Arita R, Mizoguchi T, Fukuoka S and Morishige N: Multicenter study of intense pulsed light therapy for patients with refractory meibomian gland dysfunction. Cornea 37: 1566-1571, 2018.

22. Arita R, Mizoguchi T, Fukuoka S and Morishige N: Therapeutic efficacy of intense pulsed light in patients with refractory meibomian gland dysfunction. Ocul Surf 17: 104-110, 2019.

23. Yin Y, Liu N, Gong L and Song N: Changes in the meibomian gland after exposure to intense pulsed light in meibomian gland dysfunction (MGD) patients. Curr Eye Res 43: 308-313, 2018

24. Rong B, Tang Y, Tu P, Liu R, Qiao J, Song W, Toyos R and Yan X: Intense pulsed light applied directly on eyelids combined with meibomian gland expression to treat meibomian gland dysfunction. Photomed Laser Surg 36: 326-332, 2018.

25. Rong B, Tang Y, Liu R, Tu P, Qiao J, Song W and Yan X: Long-term effects of intense pulsed light combined with meibomian gland expression in the treatment of meibomian gland dysfunction. Photomed Laser Surg 36: 562-567, 2018.

26. Jiang $\mathrm{X}$, Lv $\mathrm{H}$, Song $\mathrm{H}$, Zhang $\mathrm{M}$, Liu $\mathrm{Y}, \mathrm{Hu} \mathrm{X}$, Li $\mathrm{X}$ and Wang W: Evaluation of the safety and effectiveness of intense pulsed light in the treatment of meibomian gland dysfunction. J Ophthalmol 2016: 1910694, 2016.

27. Gupta PK, Vora GK, Matossian C, Kim M and Stinnett S: Outcomes of intense pulsed light therapy for treatment of evaporative dry eye disease. Can J Ophthalmol 51: 249-253, 2016.

28. Vegunta S, Patel D and Shen JF: Combination therapy of intense pulsed light therapy and meibomian gland expression (IPL/MGX) can improve dry eye symptoms and meibomian gland function in patients with refractory dry eye: A retrospective analysis. Cornea 35: 318-322, 2016.

29. Karaca EE, Evren Kemer Ö and Özek D: Intense regulated pulse light for the meibomian gland dysfunction. Eur J Ophthalmol 30: 289-292, 2020.

30. Seo KY, Kang SM, Ha DY, Chin HS and Jung JW: Long-term effects of intense pulsed light treatment on the ocular surface in patients with rosacea-associated meibomian gland dysfunction. Cont Lens Anterior Eye 41: 430-435, 2018.

31. Toyos R, Toyos M, Willcox J, Mulliniks H and Hoover J: Evaluation of the safety and efficacy of intense pulsed light treatment with meibomian gland expression of the upper eyelids for dry eye disease. Photobiomodul Photomed Laser Surg 37: 527-531, 2019.

32. Moher D, Shamseer L, Clarke M, Ghersi D, Liberati A, Petticrew M, Shekelle P and Stewart LA; PRISMA-P Group: Preferred reporting items for systematic review and meta-analysis protocols (PRISMA-P) 2015 statement. Syst Rev 4: 1, 2015.

33. Higgins J and Green S: Cochrane Handbook for systemic reviews of interventions. Version 5.1: The Cochrane Collaboration, 2011. https://handbook-5-1.cochrane.org. Accessed July 1, 2019.

34. Vora GK and Gupta PK: Intense pulsed light therapy for the treatment of evaporative dry eye disease. Curr Opin Ophthalmol 26: 314-318, 2015.

35. Albietz JM and Schmid KL: Intense pulsed light treatment and meibomian gland expression for moderate to advanced meibomian gland dysfunction. Clin Exp Optom 101: 23-33, 2018.

36. Ngo W, SItu P, Keir N, Korb D, Blackie C and Simpson T: Psychometric properties and validation of the standard patient evaluation of eye dryness questionnaire. Cornea 32: 1204-1210, 2013.

37. Vidas Pauk S, Petriček I, Jukić T, Popović-Suić S, Tomić M, Kalauz M, Jandroković S and Masnec S: Noninvasive tear film break-up time assessment using handheld lipid layer examination instrument. Acta Clin Croat 58: 63-71, 2019. 